\title{
COUPLED TRANSIENT ANALYSIS OF A CORE WITH FUEL ASSEMBLY BOWING WITH A HYBRID CTF/DYN3D MODEL
}

\author{
Périn Y, Travleev A, and Zilly $\mathbf{M}^{*}$ \\ Gesellschaft fuer Anlagen- und Reaktorsicherheit (GRS) gGmbH \\ Boltzmannstr. $14-85748$ Garching bei Muenchen - Germany \\ yann.perin@grs.de, anton.travleev@grs.de,matias.zilly@framatome.com
}

\begin{abstract}
Fuel assembly bowing is a known phenomenon observed in many PWR reactors all over the world. The phenomenon is relevant to safety as it can lead to increased water gaps between assemblies which results in higher pin peaking factors. The goal of the present study is to assess the effect of assembly bowing not only for stead-state nominal conditions but also during a transient. The selected transient is the loss of one reactor coolant pump as it can be limiting especially regarding the Departure from Nucleate Boiling (DNB) safety criterion. This study focuses on an extreme case where the bowing is simulated in the core hot assembly by keeping the water gap constant over the whole core active length. The resulting cross-sections and form functions obtained from a $2 \mathrm{~d}$ infinite lattice model are used in the nodal diffusion code DYN3D applying its pin-by-pin reconstruction method. For the transient simulation, DYN3D is coupled with the thermal-hydraulics subchannel code CTF on the SALOME platform. Several modelling options are compared: nominal geometry for neutronics and thermal-hydraulics (TH); mixed: neutronics with increased water gap, $\mathrm{TH}$ with nominal geometry; and increased water gap for both neutronics and TH. The results confirm that the increased water gap should be considered in both models in order to reduce the conservatism.
\end{abstract}

KEYWORDS: fuel assembly bowing, coupled simulation

\section{INTRODUCTION}

Fuel assembly bowing is a known phenomenon identified in many PWR reactors all over the world (e.g. Germany, France). The bowing can take different shapes, e.g. S-shape, W-Shape [1]. The phenomenon is relevant to safety as it can lead to increased water gaps between assemblies which results in higher pin peaking factors in the neighbouring rods due to improved moderation. It is generally assumed that the high peaking factors influence on safety parameters is (partially) compensated by the improved cooling thanks to the larger gaps.

The goal of the present study is to assess the effect of assembly bowing not only for stead-state nominal conditions but also during a transient. The selected transient is the loss of one reactor coolant pump (RCP) as it can be limiting especially regarding the Departure from Nucleate Boiling (DNB) safety criterion. This study focuses on an extreme case where the bowing is simulated in a fresh MOX assembly by keeping the a constant additional $15 \mathrm{~mm}$ to the nominal water gap over the whole core active length.

\footnotetext{
* formerly at GRS, present address: Framatome GmbH, Germany. Author's contribution during GRS employment.
} 
For this work, the capabilities of GRS' in-house core simulator KMACS [2] were extended to allow the modelling of increased (/decreased) inter-assembly water gap during the cross-section generation step as well as the assembly burnup step. Two modelling approaches, presented and compared in Section 2, were tested:

- explicit geometrical representation of the modified water gap size

- representation of the modified gap by means of the gap water density changes (selected method)

The resulting cross-sections and form functions are used in the nodal diffusion code DYN3D [3] applying its pin power reconstruction method. For the transient simulation, DYN3D is coupled with the thermalhydraulics subchannel code CTF on the SALOME platform. In CTF [4], a hybrid model is used: the core is represented at the assembly level while a pin-by-pin description of the hot assembly is applied. The models for both codes and the coupling scheme are presented in Section 3.

Finally, in Section 4, the results of the coupled simulation of the loss of one RCP transient are presented and discussed. Several modelling options are compared: nominal geometry for neutronics and thermalhydraulics $(\mathrm{TH})$; mixed: neutronics with increased water gap, $\mathrm{TH}$ with nominal geometry; and increased water gap for both neutronics and $\mathrm{TH}$.

\section{NEUTRONICS MODELING OF ASSEMBLY BOWING}

\subsection{KMACS Core Simulator}

KMACS is a classical two-step core simulator, in which the physical properties of individual fuel assembly types are first calculated in infinite lattice (so-called homogenized cross-sections), parameterized according to anticipated thermal-hydraulics states. In the second step, these fuel assemblies are simulated in the core using a nodal diffusion code and considering thermal-hydraulic feedback.

The KMACS 'KSpect' package handles the XS generation process. First the KMACS input data is converted into the lattice code input format. For this study, the NEWT [5] code from the SCALE package developed by the Oak Ridge National Laboratory (ORNL) is applied. KMACS also supervises the code execution and collects the results which are then stored in a database.

The generated cross-sections are then applied in the KMACS 'KFlux' package which computes the core flux distribution for each burnup point iteratively with the 'KBurn' depletion package. Here, DYN3D is used as 'KFlux' backend.

\subsection{Model A: Explicit Modelling of the Non-Symmetric Water Gap}

On all four sides of the lattice code model, reflective boundary conditions are assumed. According to the generalized equivalency theory [6], applied for the XS generation, the volume of the heterogeneous system, from which the homogenized XS are calculated, and the volume in which the homogenized XS are used, must be the same. Thus, local lattice changes are not directly modellable. Therefore, the use of the Model A XS in a nodal grid is not possible without corrections. In principle, corrections like the one used for the treatment of thermal expansion in liquid metal-cooled reactors [7] could be applied. However, this requires modifications in the source code of the flux solver that were not performed in this project.

\subsection{Model B: Modelling of the Non-Symmetric Water Gap Using Equivalent Water Density}

In the model of the equivalent density change, the gap is not geometrically modelled, but the effect of enhanced moderation is approximated by an increase in the water density in the gap. The total moderator 
mass in the (constant) gap of model B is the same as in model A (modified) gap. Fig. 1 shows both lattice codes models.
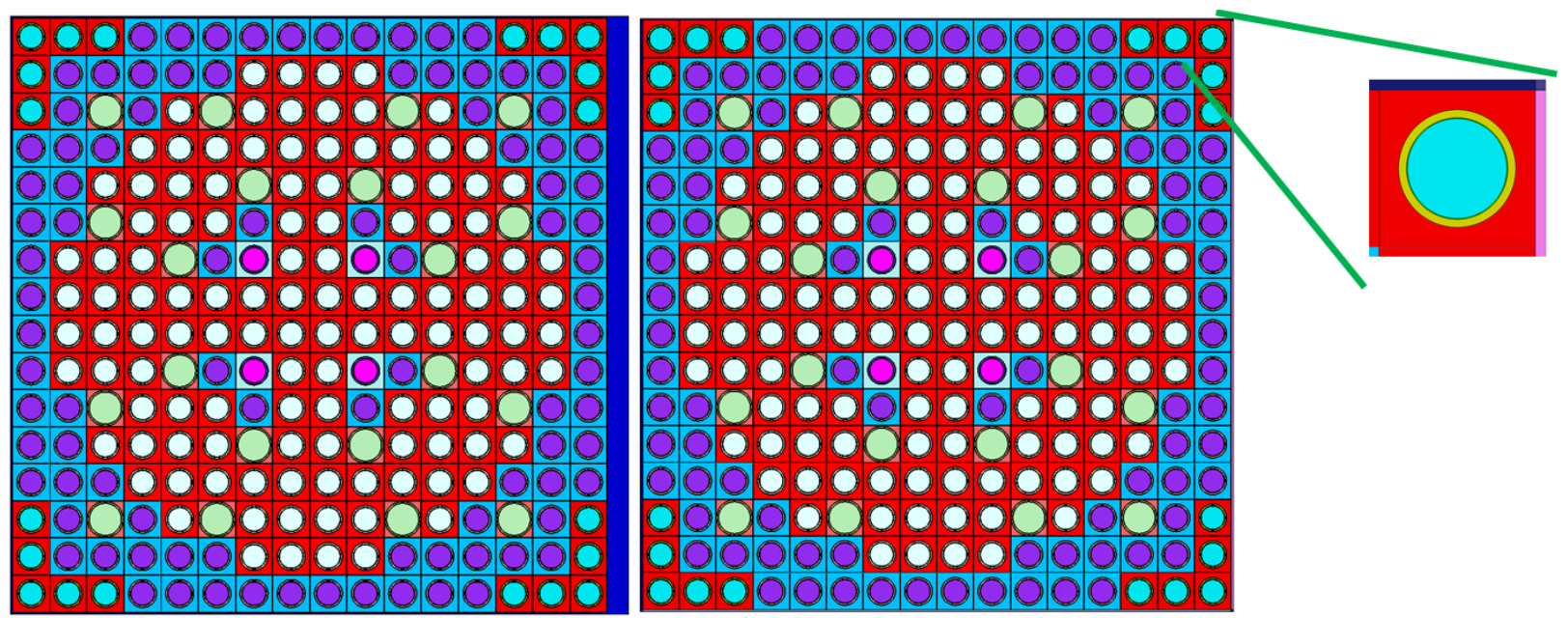

Figure 1. Lattice code models representing single FA: Left - explicit water gap modelling, Right water gap modelling through equivalent water density

The moderator density is potentially modified in eight different regions (four sides plus four corners) of the nominal gap. Figure 2 indicates that for typical gap sizes $<1 \mathrm{~cm}$, both models yield almost the same multiplication factor. Assessment with SCALE made for detailed $2 \mathrm{~d}$ models of different FAs have shown that pin power distribution obtained in both model A and model B have similar behaviour and the pin peaking factor obtained in model B differs from that predicted in model A by less than $2 \%$ for typically measured FA gap increases. Similar results were observed for MOX and UOX fuel types, for moderator densities between $620 \mathrm{~kg} / \mathrm{m}^{3}$ and $996 \mathrm{~kg} / \mathrm{m}^{3}$.

Having confirmed its validity for the intended application domain, the modelling approach B was implemented in KMACS and is applied throughout the rest of the present work.

\section{COUPLED MODELING OF ASSEMBLY BOWING}

\subsection{DYN3D Neutronics Code}

DYN3D [2] is a reactor core simulator developed at the Helmholz Zentrum Dresden Rossendorf (HZDR), Germany. It is applied for performing of steady state and transient analysis in LWR for hexagonal or square fuel assemblies' geometries. The diffusion equation is solved using a nodal expansion method. Pin-by-pin simulations are possible using either a pin power reconstruction method or the recently developed multigroup simplified transport (SP3) capabilities.

It should be pointed out that it is not currently possible to use side-dependent ADFs and pin power reconstruction at the same time, so that the results in the pin power for the calculations presented here can only be read qualitatively. 


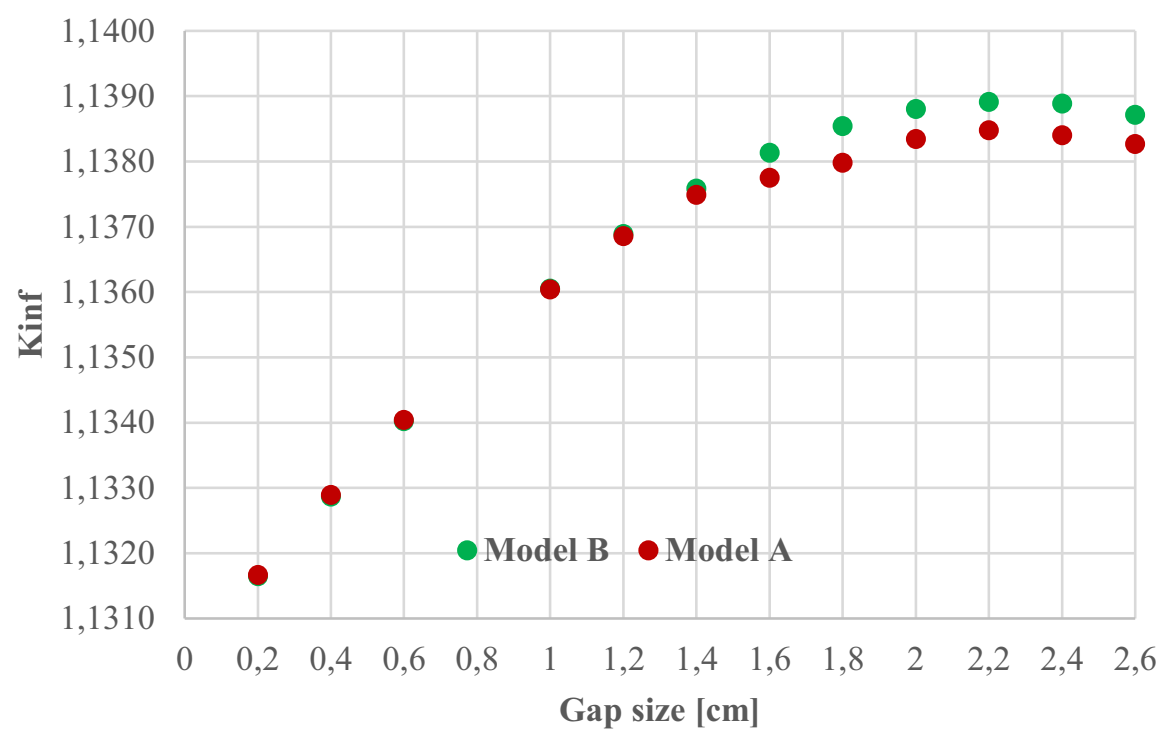

Figure 2. Multiplication factor of a MOX fuel assembly for different water gap size (moderator density $\left.=711 \mathrm{~kg} / \mathrm{m}^{3}\right)$

\subsection{DYN3D Core Model}

A simplified core loading scheme is shown in Fig. 3. The large number of different fuel assembly (FA) types has been grouped into five groups with similar enrichments, M1 and M2 are two groups of MOX-FA types, U1 and U2 are two groups of UO2-FA types, and G1 is a group of UO2-Gd-FA types. The colors blue, green, yellow and red designate FA on the 1st, 2nd, 3rd and 4th cycles.

In order to cover the anticipated reactor conditions for both the stationary and transient case, the XS are parameterized for fuel temperatures from 300 to $2500 \mathrm{~K}$, boron concentrations from 0 to $1800 \mathrm{ppm}$ and moderator densities from 620 to $996 \mathrm{~kg} / \mathrm{m}^{3}$. In addition, these combinations were varied for each of 30 burnup points as well as controlled and uncontrolled states.

In order to demonstrate the basic possibility of modelling a core with fuel assembly bending and gaining qualitative insights into the effect of bending, a very simple, unrealistic bending scenario was designed. Therein a single additional gap of $15 \mathrm{~mm}$ width was inserted between the FAs at positions L7 and L8. This additional gap was assumed constant over the entire axial length of the core, so that a quasi-two-dimensional model is suitable for the description. To compensate for the additional gap, the gaps between the FAs at positions L4 to L11 are closed, and the gaps between L3 and L4 and between L11 and L12 are reduced. In practice, this means that individual cross-section libraries (and form functions) were generated with KMACS for each of the assembly in row ' $L$ ' using the equivalent water density approach described in Section 2.

As a reference, a XS library generated using only the nominal water gaps is also used for comparison purposes. 


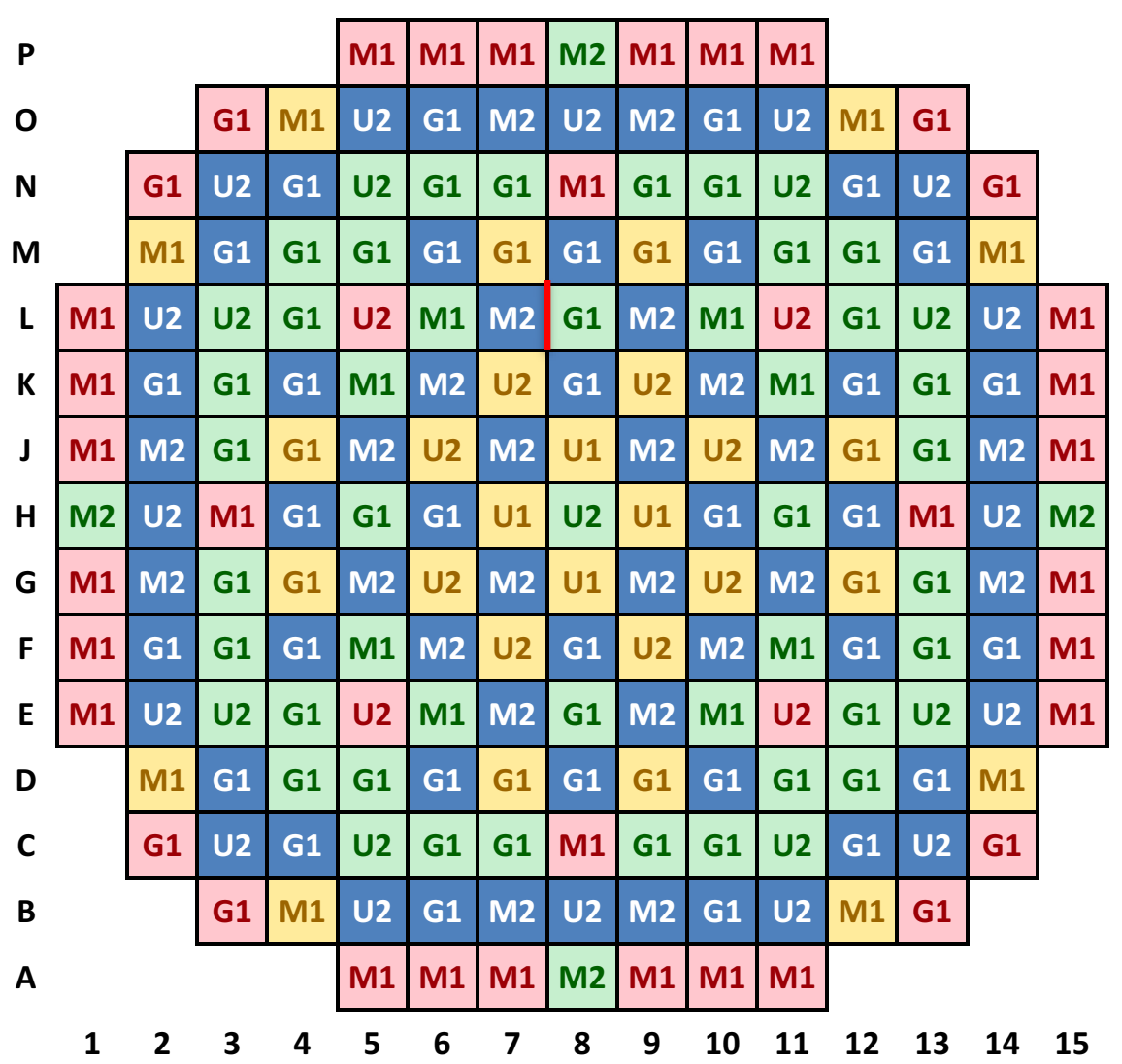

Figure 3. Core loading. The increased water gap between $\mathrm{L} 7$ and $\mathrm{L8}$ is marked in red

\subsection{CTF Thermal-Hydraulics Sub-Channel Code}

Coolant-Boiling in Rod Arrays | Two Fluids (COBRA-TF) is a modern 3D Thermal/Hydraulic Sub-Channel simulation code designed for LWR analysis [3]. It has been improved and updated at the North Carolina State University (NCSU), USA by the Reactor Dynamics and Fuel Management Group (RDFMG) and subsequently re-branded as CTF.

\subsection{CTF Model}

The core model in CTF is a so-called hybrid model: each fuel assembly is modelled with a single channel, and only the two fuel assemblies L7 and L8 with the increased water gap are modelled pin-by-pin. With this hybrid approach, the model size and computational time remains tractable, while the safety parameter can be calculated at the pin level. All channels are so-called "pin-centered" channels, which are not optimal for thermo-hydraulic modelling, but allow the data exchange for the coupled calculation. The model features:

- Assembly-wise part of the active core: 191 channels each one containing a fuel rod object with a multiplier (232 for MOX fuel and 236 for all other types),

- 56 channels for the radial reflector,

- 2x256 channels in the MOX fuel assembly (L7) and UO2-Gd fuel assembly (L8),

- 232 individual fuel rods in the MOX fuel assembly (L7),

- 236 individual fuel rods in the UO2-Gd fuel assembly (L8),

- 17 nodes in the axial direction (same nodalization as in DYN3D). 
Two version of this model are built, one with the nominal water gap between L7 and L8 and one with the modified water gap.

\subsection{Coupling of DYN3D and CTF on the SALOME Platform}

The SALOME platform [8] is an open-source software codeveloped by EDF, CEA and OpenCascade. Originally created for CAD applications, it has since evolved into a platform for code coupling in the framework of a series of three consecutive European Commission founded projects: NURESIM (20062008), NURISP (2009-2011) and NURESAFE (2013-2015). In the framework of the NURESAFE project [9] the subchannel code CTF and the neutronics code DYN3D were integrated and coupled on the Salomé platform. Coupling on the SALOME platform is compatible with CTF hybrid models are DYN3D pin power reconstruction method [10].

\section{COUPLED TRANSIENT ANALYSIS}

\subsection{Loss of one Reactor Coolant Pump Transient}

The selected transient is the failure of one RCP. The loss of one RCP transient can lead to a decrease of the DNB ratio because less coolant flows through the core shortly after the RCP stops while at the same time the power remains high.

When the speed of a main coolant pump drops below a certain system-specific level (selected here: 94\%), the control unit automatically reduces the reactor output due to control rod insertion. From this point on, the DNBR increases again which is why this study focuses on the first 10 s of the transient.

The thermohydraulic boundary conditions at the core inlet and outlet were extracted from an existing loss of RCP simulation with a system code and implemented in CTF.

\subsection{Results of the DYN3D/CTF Simulations}

The next paragraph presents the coupled results of the loss of RCP transient with CTF / DYN3D. Several combinations of both models are possible and are shown in Table I. Only the most relevant combinations are presented in the next paragraphs.

Table I. DYN3D-CTF Model combinations

\begin{tabular}{|c|c|c|c|c|}
\hline $\begin{array}{l}\text { DYN3D } \rightarrow \\
\text { CTF } \downarrow\end{array}$ & $\begin{array}{c}\text { Nominal } \\
\text { geometry }\end{array}$ & $\begin{array}{c}\text { Modified } \\
\text { water gap }\end{array}$ & $\begin{array}{c}\text { Nominal geometry + } \\
\text { Pin power reconstruction }\end{array}$ & $\begin{array}{c}\text { Modified water gap + } \\
\text { Pin power reconstruction }\end{array}$ \\
\hline $\begin{array}{c}\text { Nominal } \\
\text { geometry }\end{array}$ & Model 1 & Model 2 & N/A & Model 3 \\
\hline $\begin{array}{c}\text { Modified } \\
\text { water gap }\end{array}$ & N/A & N/A & N/A & Model 4 \\
\hline
\end{tabular}

\subsubsection{Results of model 1 and 2 simulations}

Fig. 4 shows the minimum DNB ratio in models 1 and 2 during the loss of RCP transient. Before transients, the minimum value of this ratio is significantly lower in model 2 at 1.75 compared to 2.31 in model 1 . When the control rods are inserted, the DNB ratio increases rapidly. Previously, a minimum is achieved: 1.73 in model 2 and 2.29 in model 1. 


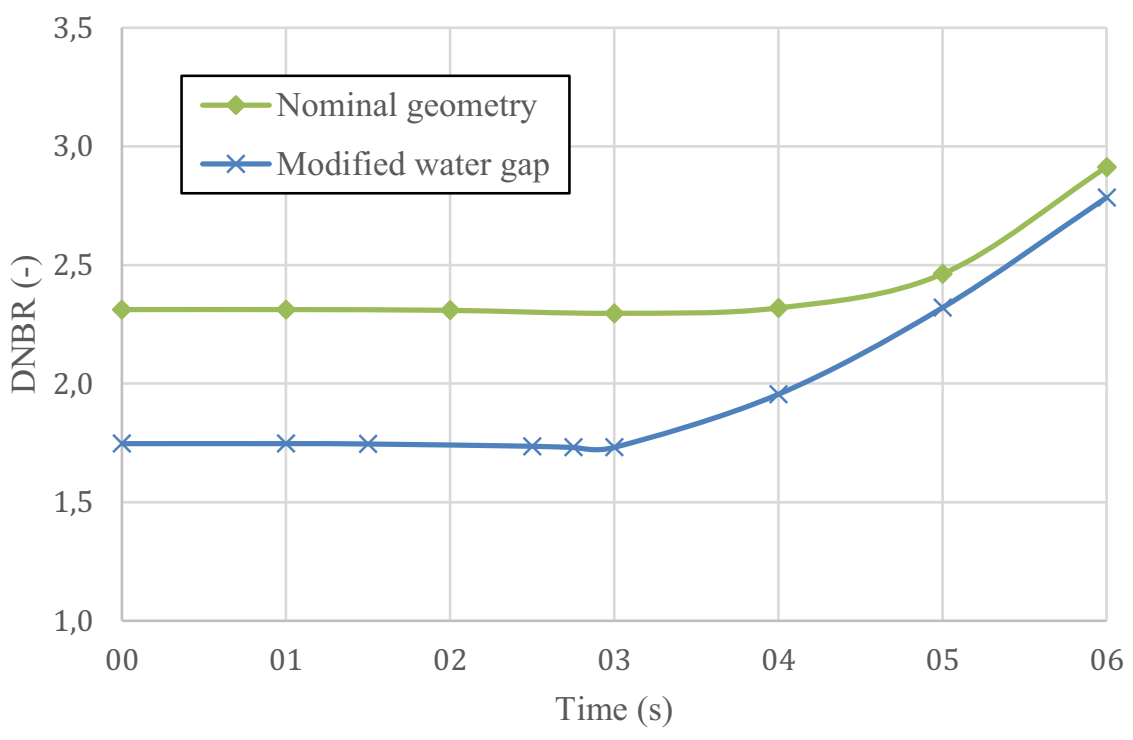

Figure 4. Comparison of the DNB Ratio during a loss of RCP transient for models 1 and 2

\subsubsection{Results of model 3 and 4 simulations}

For these models, however, the coupled system CTF / DYN3D could not converge. Therefore, no transient simulation could be performed. The last converged iteration of the pin power distribution from DYN3D was therefore inserted in a CTF stand-alone model. For model 3 , the DNB is reached at 0.54 , and the code cannot converge. For Model 4, the minimum DNB ratio is 0.97. As expected, the DNB parameter is higher with thermo-hydraulic modelling of the water gap. In this case, the improved cooling cannot compensate for the (unrealistic) high power density of DYN3D.

\section{CONCLUSIONS}

For this work, the capabilities of GRS' in-house core simulator KMACS were extended to allow the modelling of increased (/decreased) inter-assembly water gap during the cross-section generation step as well as the assembly burnup step. Two modelling approaches were tested:

- explicit geometrical representation of the modified water gap size

- representation of the modified gap by means of the gap water density changes

Both methods were compared and show a very good agreement. The second approach was finally chosen as its implementation in the nodal diffusion code does not require any changes in the model geometry.

Finally, this core model was used for the simulation of a reactor transient. For this purpose, the failure of a main coolant pump was simulated with the coupled code system CTF / DYN3D. CTF is a subchannel code for modelling rod-level thermo-hydraulics. The loss of one RCP transient was selected as it can be limiting regarding the DNB ratio safety criterion. The boundary conditions at the core inlet and exit as well as the control rod positions were extracted from an existing system code calculation and applied to the CTF / DYN3D models. The essential results are:

- The DNB ratio decreases when an increased inter-assembly water gap is modelled neutronphysically (in DYN3D), 
- Explicit thermo-hydraulic modelling of the increased water gap (in CTF) increases the value of DNB ratio through improved cooling.

When the pin power reconstruction is performed with DYN3D, very high (unrealistic) pin powers are calculated which cause the critical DNB ratio to fall short. To avoid this problem, simulations of more realistic fuel bending scenarios should be performed in the future.

For future work, other transients of interest include control rod ejection and ATWS. Furthermore, the influence of "pin-centered" and "coolant-centered" modelling in CTF on the results of thermo-hydraulics will be investigated. Indeed, "pin-centered" channels allow a straightforward data exchange for the coupled calculations but are not optimal for thermo-hydraulic modelling. The influence of the coolant-centered modelling will be interesting especially for the DNBR estimation. Finally. for a more realistic modelling, the thermo-hydraulic feedback on the individual fuel power should also be investigated (high-fidelity coupling).

\title{
NOMENCLATURE
}

\author{
CTF COBRA-TF \\ DNB Departure from Nucleate Boiling \\ FA Fuel Assembly \\ PWR Pressurized Water Reactor \\ RCP Reactor Coolant Pump
}

\section{ACKNOWLEDGMENTS}

This work was supported by the German Federal Ministry for the Environment, Nature Conservation and Nuclear Safety.

\section{REFERENCES}

1. J. Berger, Impact of fuel assembly bowing on the power density distribution and its monitoring in Siemens/KWU-PWR, Master's Thesis, KTH, Stockholm, 2017

2. J. Bousquet, M. Zilly and M. Küntzel, KMACS User Manual, 2018, https://www.grs.de/kmacs

3. U. Rhode et al., "The reactor dynamics code DYN3D - models, validation and applications", Progress in Nuclear Energy, 89, 170-190 (2016)

4. M. Avramova et al., CTF User's Manual, Technical report, Reactor Dynamics and Fuel Modeling Group, The North Carolina State University

5. B. T. Rearden and M. A. Jessee, Eds., SCALE Code System, ORNL/TM-2005/39, Version 6.2, Oak Ridge National Laboratory, Oak Ridge, Tennessee (2016). Available from Radiation Safety Information Computational Center as CCC-834

6. K.S. Smith, “Assembly Homogenization Techniques for Light Water Reactor Analysis", Progress in Nuclear Energy, 17, 303 (1986)

7. J. Bousquet, A. Seubert, P. Sarkadi, "3-D Coupled PARCS/ATHLET Simulation of SFR Using an Explicit Radial Expansion Model”, Proceedings of the PHYSOR 2018, Cancún, 22.-26. April 2018

8. SALOME version 9.3.0 Release Notes, May 2019, https://www.salome-platform.org/

9. B. Chanaron, "7th Framework Programme - Simulation Platform for Nuclear Reactor Safety - Grant agreement for: Collaborative project - Annex I - Description of Work," Technical report (2012).

10. Y. Périn, K. Velkov, "CTF / DYN3D Multi-Scale Coupled Simulation of a Rod Ejection Transient on the Salomé Platform", Proceedings of the M\&C 2017, Jeju, Korea, April 16-20, 2017 